\title{
Noncompensatory consideration and compensatory choice: an application to Stackelberg competition
}

\author{
Mauro Papi
}

Received: 13 November 2013 / Accepted: 20 December 2013 / Published online: 7 January 2014 (C) SAET 2014

\begin{abstract}
Unlike standard models of choice, experimental evidence suggests that individuals avoid tradeoffs when choice problems are complex. I analyze the implications of consumers using noncompensatory choice heuristics in a simple Stackelberg game in which firms offer menus of multi-attribute alternatives and influence the attribute considered to be salient by consumers via marketing. I illustrate that there is a tight link between optimal menu design and marketing strategies in equilibrium and briefly discuss the choice-theoretic properties of the consumer's choice procedure.
\end{abstract}

Keywords Bounded rationality · Compensatory ·

Noncompensatory choice heuristics · Stackelberg

JEL Classification $\quad \mathrm{D} 00 \cdot \mathrm{L} 00$

\section{Introduction}

Standard models of choice assume that the decision-maker (DM) makes tradeoffs independently of the characteristics of the choice problem. Over the last two decades experimental evidence has challenged this paradigm: contrary to what standard models

I would like to thank Ran Spiegler for his helpful suggestions. I am also grateful to Miguel Angel Ballester, Johannes Hoerner, Philippe Jehiel, Erika Magnago, Paola Manzini, Marco Mariotti, William Neilson, an anonymous referee, the editor Nicholas C. Yannelis, and the seminar audience at Aberdeen and UCL for their comments. Any error is my own responsibility.

M. Papi $(\bowtie)$

Business School, University of Aberdeen, Edward Wright Building,

Dunbar Street, Old Aberdeen AB24 3QY, UK

e-mail: m.papi@abdn.ac.uk 
assume, the extent to which the DM is able to make tradeoffs crucially depends on the choice environment (e.g., number of alternatives, amount of time pressure, etc.).

Payne et al. (1993) define a choice heuristic to be compensatory (resp., noncompensatory) whenever the DM makes (resp., does not make) tradeoffs between attributes. Experiments typically suggest that whenever the choice problem is relatively simple, DMs tend to follow decision strategies, such as compensatory ones, that are accurate, but require relatively high cognitive effort. On the contrary, whenever the choice problem gets complex, they sacrifice accuracy by relying on decision strategies, such as noncompensatory ones, that require relatively little cognitive effort. ${ }^{1}$

In this note I study a simple multi-attribute market model in which consumers simplify complex problems by discarding all alternatives that do not possess some salient attribute (noncompensatory phase) and then choose by maximizing an utility function among the alternatives that survive (compensatory phase), if any. Consistently with the experimental evidence, I assume that at simple problems consumers use a compensatory choice heuristic (i.e., utility maximization) straight away and identify the complexity of a choice problem with its cardinality. Firms compete à la Stackelberg by offering menus of multi-attribute alternatives and influencing the attribute that the consumer considers to be salient in the noncompensatory phase via marketing.

I find that there is a tight link between the optimal menu design and marketing in equilibrium. If firms face technological constraints (i.e., only alternatives that possess up to a certain number of attributes can be offered), then in any equilibrium the leader uses product differentiation as an entry deterrence and marketing is irrelevant. On the contrary, in any equilibrium under fixed-capital constraints (i.e., only alternatives that possess certain specific attributes can be offered), both firms are active in the market and whenever the consumer goes through the noncompensatory phase marketing is not only relevant but also firm-specific, and firms offer menus accordingly. Finally, I briefly discuss the choice-theoretic properties of the consumer's choice procedure and examine its relationships with other models.

The paper is organized as follows: Sect. 2 defines the game; Sects. 3 and 4 propose the equilibrium analysis; Sect. 5 examines the consumer's choice procedure in detail; Sect. 6 discusses the related literature, limitations, and extensions. Proofs are relegated in the Appendix.

\section{A Stackelberg game}

I assume that two firms, a leader (L) and a follower (F), compete to maximize profits in a Stackelberg game. L moves first, F observes what L does and then chooses a strategy. Each firm's strategy is a pair $(M, m)$, where $M \in 2^{\{0,1\}^{k}} \backslash \emptyset$ is a menu of multi-attribute alternatives and $m \in\{1, \ldots, k\}$ the attribute that is advertised. I assume attributes to be binary and interpret alternative $x \in M$ possessing (resp., not possessing) attribute $i$ whenever $x_{i}=1$ (resp., $x_{i}=0$ ). The $\operatorname{cost} c(M, m) \equiv \kappa \sum_{x \in M} \sum_{i \in\{1, \ldots, k\}} x_{i}$ of offering $(M, m)$ is given by the number of supplied attributes times a positive constant $\kappa>0$ and marketing is costless. A sold product yields a fixed level of revenues

\footnotetext{
${ }^{1}$ See for example Payne et al. (1993), Hauser et al. (2009), Gigerenzer et al. (2011).
} 
normalized to $£ 1$. I assume that attributes are good and interpret a competitor offering $(\{(0, \ldots, 0)\}, a)$ as it not being active in the market.

On the demand side, there is a population of consumers of measure 1 endowed with a utility function $u(x) \equiv \sum_{i} \alpha_{i} x_{i}$, where $\infty>\alpha_{i}>0$. I allow for preference heterogeneity (different types can have different $\alpha \mathrm{s}$ ) and denote by $u \in U$ a consumer's type in the set of consumer's types. I assume that if the consumer's feasible set is simple, where by simple I mean that its cardinality is below a threshold normalized to two, then type $u$ uses a compensatory choice heuristic by choosing the $u$-highest utility alternative available. On the contrary, if the feasible set is complex (i.e., its cardinality is greater than two), then type $u$ first uses a noncompensatory choice heuristic by eliminating all alternatives that do not possess an attribute considered to be salient (e.g., a low-fat yogurt, a high-speed broadband, a holiday on the Red Sea, etc.). Second, she chooses the $u$-highest utility alternative available among those that survive (if any). I assume that choosing nothing is always worse than choosing something in terms of utility. On the path of play $\left[\left(M_{L}, m_{L}\right),\left(M_{F}, m_{F}\right)\right]$, the consumer's feasible set is $M_{L} \cup M_{F}$ and her salient attribute is endogenously determined in the following way. The salient attribute is $m$, if both firms advertise $m$ (i.e., $m=m_{L}=m_{F}$ ), and $m_{F}$ and $m_{L}$ with equal probability, if the marketing messages are distinct (i.e., $m_{L} \neq m_{F}$ ). Hence, if firms use distinct marketing strategies, then there are two equally likely states of the world: one in which the consumer's salient attribute is $m_{L}$ and the other in which it is $m_{F}$. Everything is common knowledge and ties are broken in favour of the first mover.

\section{Technological constraints}

I say that menu $M$ is feasible under technological constraints whenever $M \subseteq\{x \in$ $\left.\{0,1\}^{k}: \sum_{i} x_{i} \leq h\right\}$ for some $h \leq k$. That is, a menu of products is feasible if and only if it possesses up to $h \leq k$ attributes, where $k$ is the maximum number of attributes a product can have.

As an example assume that products are tyres and are characterized by three attributes: winter, summer, and grip. It is technologically impossible to produce a tyre that is suitable for both winter and summer and has an excellent grip. A tyre can either be suitable for winter and have an excellent grip, or be suitable for summer and have an excellent grip, or be usable all over the year and not have an excellent grip in neither winter nor summer.

Assume that $k=3$ and $h=2$. Suppose that there are two consumer's types $u$ and $v$ that occur with probability $q>\frac{1}{2}$ and $1-q$ such that $u(011)>u(101)>u(110)$ and $v(110)>v(011)>v(101)$, respectively. If the consumer is fully rational, then marketing is irrelevant and, as long as costs are sufficiently small, L offers a menu $\left(\{(011),(110)\}, m_{L}\right)$ that contains a top utility alternative for every type and grabs the whole market. Is this still an L's equilibrium strategy if the consumer is boundedly rational?

Assume that the consumer is boundedly rational. Then, F's best response to $\mathrm{L}$ offering $\left(\{(011),(110)\}, m_{L}\right)$ is $(\{(101)\}, 1)$. This strategy yields to $\mathrm{F}$ at least $\frac{q}{2}$ market shares at a cost of $2 \kappa$. The reason is that the feasible set has cardinality three 
implying that the consumer goes through the noncompensatory phase. In at least one half of the cases the consumer's salient attribute is 1 and (101) is the highest utility alternative that possesses attribute 1 with probability $q$. Note that, by offering ( $\left.(011),(110),(101)\}, m_{L}\right)$, L grabs the whole market, because this menu contains all $u$ - and all $v$-highest utility products that possess attribute $i$ for any $i$. This neutralizes F's marketing, prevents $F$ from entering the market and, if costs are sufficiently small, constitutes an L's equilibrium strategy.

Proposition 1 (Technological Constraints) Assume that firms face technological constraints and costs are sufficiently small. Then, in any equilibrium on the path of play $M_{L}^{*}=\bigcup_{u \in U}\left\{x \in \arg \max _{y \in\{0,1\}^{k}} u(y): x_{i}>0 \forall i\right\}, M_{F}^{*}=\{0\}$, and marketing is irrelevant.

Proposition 1 generalizes the result of the above example by suggesting that $\mathrm{L}$ can prevent $\mathrm{F}$ from entering the market by offering a menu that contains a $u$-top utility alternative that possesses attribute $i$ for any $i$ for any type $u$. As highlighted above, the model predicts more product differentiation relative to the case in which the consumer is fully rational. In particular products that do not yield the maximal utility are offered with positive probability. The reason is that products that yield the highest utility among those that possess some attribute $i$ can be chosen. Therefore, if $\mathrm{L}$ does not offer all those products, whether inferior or not, then F can enter the market and grab some market shares. Thus, as long as costs are sufficiently small, L adopts product differentiation as an entry deterrence and makes marketing irrelevant.

\section{Fixed-capital constraints}

I say that $M$ is feasible under fixed-capital constraints whenever there exists a set $\hat{I}_{M}$ of $l<k$ attributes such that $M \subseteq\left\{x \in\{0,1\}^{k}:\left\{i: x_{i}>0\right\} \cap \hat{I}_{M}=\emptyset\right\}$. That is, firms are constrained in terms of machinery as they cannot offer products that possess a set $\hat{I}_{M}$ of $l$ specific attributes.

Getting back to the tyre example, it might be the case that a firm possesses the machinery to produce only high-quality winter tyres, because acquiring the fixedcapital needed to produce summer tyres might be too costly, though technologically feasible. Alternatively, assume that alternatives are bottles of whisky characterized by three attributes, such as whether the whisky is smoked, spicy, and fruity or not. It might be the case that a distillery $A$ cannot produce a spicy whisky (but only a smoked and/or fruity one), because the technology to do so is known only to distillery $B$.

I look for the equilibrium under the assumption that firms by offering a menu $M$ implicitly select their fixed-capital. That is, firms implicitly choose the set $\hat{I}_{M}$ of attributes that they cannot produce. ${ }^{2}$ The interpretation consistent with this analysis is that firms have a budget that they can spend in acquiring the fixed-capital needed to produce alternatives that possess $k-l$ specific attributes. The choice of $I_{M}$ will depend on the distribution of types and their preferences.

\footnotetext{
${ }^{2}$ In the case in which fixed-capital constraints are exogenously given, the two versions (technological and fixed-capital constraints) of the model differ whenever $\hat{I}_{M_{L}} \neq \hat{I}_{M_{F}}$. If, on the contrary, $\hat{I}_{M_{L}}=\hat{I}_{M_{F}}$, then firms face technological constraints such that products can possess at most $k-l$ attributes.
} 
Proposition 2 (Fixed-capital Constraints) Assume that firms face fixed-capital constraints and costs are sufficiently small. Then, in any equilibrium both firms are active in the market and whenever the consumer goes through the noncompensatory phase marketing is relevant and firm-specific.

To fix ideas reconsider the example I have discussed in the previous section. That is, assume that there are two consumer's types $u$ and $v$ that occur with probability $q>\frac{1}{2}$ and $1-q$ such that $u(011)>u(101)>u(110)$ and $v(110)>v(011)>v(101)$, respectively. In addition assume that $l=1$, which means that firms cannot offer products that possess one specific attribute. If the consumer is fully rational, then $\mathrm{L}$ serves the most frequent type by offering (011), F the other type by offering (110), and marketing is irrelevant.

Assume that the consumer is boundedly rational. Suppose first that $m_{L}=1$. Then L's machinery $\hat{I}_{M_{L}}$ is either $\{2\}$ or $\{3\}$. If $\mathrm{L}$ selects machinery $\{2\}$ (resp., $\{3\}$ ), then $\mathrm{L}$ offers (101) (resp., (110)), a highest utility alternative that possesses attribute 1 with probability $q$ (resp., $1-q$ ). Since $q>1-q$, then the 'optimal machinery' is $\{2\}$. Hence, in this case L offers $(\{(101)\}, 1)$ and F best responds with $(\{(110),(100)\}, 2)$ yielding a profit of $\frac{q}{2}-2 \kappa$ to L, where (100) is a cheapest alternative whose only purpose is to make the feasible set complex. Note that by adding products to menu $\{(101)\}$, L does not gain market shares, but costs increase.

By similar arguments, when $m_{L}=2$, the optimal machinery is $\{1\}, L$ offers (011), F best responds with $(\{(110),(100)\}, 1)$, and L obtains $\frac{q}{2}-2 \kappa$ and when $m_{L}=3$, the optimal machinery is $\{1\}, L$ offers $(011), F$ best responds with ( $\{(101),(100)\}, 1)$, and L obtains $\frac{1}{2}-2 \kappa$. The marketing strategy that maximizes L's profits is $m_{L}=3$. Therefore, the equilibrium strategies on the path of play are $(\{(011)\}, 3)$ and $(\{(101),(100)\}, 1)$ for L and F, respectively. ${ }^{3}$

Interestingly, unlike technological constraints, fixed-capital constraints imply that not only both firms are active in the market but also marketing is strategically relevant and firm-specific in equilibrium whenever consumers go through the noncompensatory phase. In particular, while L uses positive marketing, F adopts negative marketing. That is, on the one hand, $L$ decides to advertise attribute $m_{L}$ only because its products possess that attribute in order to maximize the probability that its products are chosen when the consumer's salient attribute is $m_{L}$. On the other hand, $\mathrm{F}$ chooses to advertise an attribute among those that the products offered by $L$ do not have and offers a menu accordingly.

\section{Choice-theoretic properties of the consumer's choice procedure}

Let $X \subseteq\{0,1\}^{k}$ be the grand set of alternatives, with $2<k<\infty$. Let $I \equiv\{1, \ldots, k\}$ be the set of attributes. Denote by $I(x) \equiv\left\{i \in I \mid x_{i}>0\right\}$ the set of attributes that $x$ possesses. Let $\mathcal{D} \subseteq 2^{X} \backslash \emptyset$ be a choice domain. I denote a choice problem by $A \in \mathcal{D}$. I assume that the set $\left\{A \in 2^{X} \backslash \emptyset:|A|=2\right\}$ of binary choice problems to be a subset of $\mathcal{D}$. A choice function maps $\mathcal{D}$ into $X \cup\{\emptyset\}$ with the property that $c(A) \in A \cup\{\emptyset\}$.

\footnotetext{
$\overline{{ }^{3} \text { F's best response to L offering }}(\{(011)\}, 3)$ is also $(\{(110),(100)\}, 1)$, which leads exactly to the same outcome.
} 
Given $A \in \mathcal{D}$, let $A(i)$ be defined as $\{y \in A \mid i \in I(y)\}$ if $\{y \in A \mid i \in I(y)\} \neq \emptyset$ and $\{\varnothing\}$, otherwise. That is, $A(i)$ denotes the set of alternatives in $A$ that possess attribute $i$. Let $U C_{x, A} \equiv\{y \in A \mid y=c(\{x, y\})\}$ be the set of alternatives in $A$ that beat $x$ in pairwise comparison.

Consider the following axioms.

A1 (binary transitivity): if $x=c(\{x, y\})$ and $y=c(\{y, z\})$, then $z=c(\{x, z\})$.

A2 (binary Pareto): if $I(x) \subset I(y)$, then $y=c(\{x, y\})$.

A3 (binary nonemptyness): $c(A) \neq \emptyset$ for any $A \in \mathcal{D}$ such that $|A| \leq 2$.

A4: let $|A|>2$. If $c(A)=\emptyset$, then $A(i)=\{\emptyset\}$ for some $i \in I$.

A5: let $x \in A$ and $|A|>2$. If $A(i) \cap U C_{x, A} \neq \emptyset$ for any $i \in I(x)$, then $c(A) \neq x$.

Axioms A1-A3 are standard properties. A4 requires that a necessary condition for the choice function to be empty is that there must be an attribute that all alternatives available do not possess. A5 says that an alternative $x$ has to be discarded if for any attribute $i$ that $x$ possesses there is another alternative $y$ that possesses attribute $i$ and is chosen over $x$ in pairwise comparison.

Proposition 3 (Axiomatic Characterization of the Consumer's Choice Procedure) $A$ choice function $c$ satisfies A1-A5 if and only if there exist a strictly increasing utility function $u:\{0,1\}^{k} \cup\{\emptyset\} \rightarrow \Re$ such that $I(y) \subset I(x)$ implies $u(x)>u(y)$ and $\{\emptyset\}=\arg \min _{y \in X \cup\{\emptyset\}} u(y)$, and, for any $A \in \mathcal{D}$, an attribute $i_{A} \in I$ such that

$$
\{c(A)\} \equiv \arg \max _{x \in \Phi(A)} u(x)
$$

where

$$
\Phi(A) \equiv \begin{cases}A & \text { if }|A| \leq 2 \\ A\left(i_{A}\right) & \text { if }|A|>2\end{cases}
$$

When the above occurs we say that $c$ is a choice with limited tradeoffs (CLT). ${ }^{4}$

The consumer's choice procedure clearly violates WARP, as it might be the case that $x$ is chosen over $y$ in pairwise comparison, but $y$ is chosen at the more complex problem $\{x, y, z\}$ because $y$ is the best alternative that possesses a certain attribute. ${ }^{5}$ Does then CLT incorporate the standard model as a special case?

Proposition 4 (Relationships with the Standard Model) Assume that the choice domain $\mathcal{D}$ contains all subsets of the grand set of alternatives up to three elements, the choice function is never empty, and attributes are good. Let CLT* be a CLT such that, for any $A \in \mathcal{D}, i_{A} \in I\left(x^{* A}\right)$, where $x^{* A} \in \arg \max _{y \in A \cup\{\emptyset\}} u(y)$. Then, $c$ is an $C L T^{*}$ if and only if it satisfies WARP.

If the salient attribute is 'optimally' selected (i.e., it is possessed by the highest utility alternative at any choice problem), then the resulting CLT is equivalent to a standard maximization procedure.

\footnotetext{
${ }^{4}$ In the Appendix show that the axioms are independent.

5 WARP: let $x, y \in A \cap B$. If $x=c(A)$, then $y \neq c(B)$.
} 
In the remaining of this section I discuss two well-known models of boundedly rational choice. Manzini and Mariotti (2007) propose a model in which the DM makes a decision by sequentially applying two binary relations according to a fixed ordering. On the one hand, unlike my model, Manzini and Mariotti (2007) can explain binary cycles. On the other hand, my model violates their axioms (e.g. WWARP: let $\{x, y\} \subset A \subset B$, if $x=c(\{x, y\})=c(B)$, then $y \neq c(A))$ implying that the two models do not contain one another.

Masatlioglu et al. (2012) assume that the DM chooses her most preferred alternative among those considered by imposing that the set of considered alternatives, which they call 'attention filter', does not change if an alternative that does not attract attention is removed. Unlike Masatlioglu et al. (2012), my model cannot explain binary cycles. However, my model violates their restriction imposed on the attention filter. Hence, the two models are different.

\section{Related literature and extensions}

The paper is related to the literature on bounded rationality and industrial organization. ${ }^{6}$ Both Bordalo et al. (2013) and Dahremoeller and Fels (2013) propose a multiattribute model in which consumers may devote more attention to an attribute relative to another, attributes are non-binary, and salience is endogenous. Unlike my model, Bordalo et al. (2013) assume that the consumer's consideration set always coincides with the feasible set and the salience of a product's attribute is an increasing function of the relative distance between its value and the average of that attribute value across feasible products. On the contrary, in my model the consideration set is endogenous and salience is determined by marketing, which is a separate firm's decision. On the other hand, while Dahremoeller and Fels (2013) investigate a monopoly, I examine a duopoly.

Eliaz and Spiegler (2011) propose a market model in which firms compete on quality and can influence the consumer's consideration set via costly marketing messages. ${ }^{7}$ Piccione and Spiegler (2012), on the other hand, extend Bertrand competition by assuming that firms can influence the extent to which the DM is able to compare alternatives by putting in practice some costless marketing device. ${ }^{8}$ Unlike these models, mine investigates a sequential game and assumes that marketing influences the noncompensatory phase of the decision process.

My work could be extended in multiple ways. First, the assumption that attributes are binary is clearly a limitation of this work. In the real world, attributes can be both binary and non-binary, the most prominent non-binary attribute being the price. The fact that interesting results can be obtained out of a simple binary world suggests that an extension to a binary/non-binary framework might be promising as well.

\footnotetext{
6 As an example see Spiegler (2012). Recent surveys of this literature are Ellison (2006), Armstrong (2008), and Spiegler (2011).

7 Choice-theoretic studies on the consideration set are, for example, Eliaz et al. (2011) and the already discussed Manzini and Mariotti (2007) and Masatlioglu et al. (2012).

8 See Spiegler (2013) for an extension.
} 
Second, I identify complexity with cardinality, which I believe is a reasonable assumption. However, a more realistic definition of complexity would need to take into account other dimensions, such as the number of attributes, the amount of time pressure, and the extent to which the consumer is familiar with the products under consideration. Moreover, I normalize the threshold that defines a choice problem to be complex to two. Unlike other modifications of the model, increasing the threshold would not change the results qualitatively, as it would simply imply that firms have to offer more complicated menus to make consumers go through the noncompensatory phase.

Third, I assume that all consumer types react in the same way to a marketing strategy. It might be the case that different types with different utility functions react differently depending on the extent to which the advertised attribute is important in the utility function.

Fourth, I study a sequential game, which captures many reasonable situations (e.g., incumbent vs challenging firm). An interesting question would be to study the same game under the assumption that firms move simultaneously. I leave these extensions for future research.

\section{Appendix Proofs}

Proposition 1 Assume that firms can offer products that possess up to $h \leq k$ attributes and costs are sufficiently small. Assume that $M_{L}^{*}=\bigcup_{u \in U}\left\{x \in \arg \max _{y \in\{0,1\}^{k}} u(y)\right.$ : $\left.x_{i}>0 \forall i\right\}, M_{F}^{*}=\{0\}$, and marketing is anything. On this path of play L obtains $1-\kappa \sum_{x \in M_{L}^{*}} \sum_{i \in\{1, \ldots, k\}} x_{i} . F$ cannot profitable deviate. Assume that $L$ deviates by offering $M_{L}^{*} \backslash\left\{x^{\prime}\right\}$ for some $x^{\prime} \in M_{L}^{*}$. Since $x^{\prime} \in M_{L}^{*}$, then $x^{\prime}$ is the $u$ highest utility alternative that possesses attribute $i$ for some $u \in U$ and some $i \in\{1, \ldots, k\}$. Then, $F$ 's best response is to offer $\left(\left\{x^{\prime}\right\}, i\right)$. This deviation yields $1-A-$ $\kappa \sum_{x \in M_{L}^{*} \backslash\left\{x^{\prime}\right\}} \sum_{i \in\{1, \ldots, k\}} x_{i}$ to L, where $A \in\left\{\frac{\operatorname{Prob}(\text { type } u)}{2}\right.$, Prob(type u) $\}$ depending on whether $m_{L}^{*} \neq i$ or not, respectively. The assumption that costs are sufficiently small ensures that the deviation is not profitable, a contradiction. Adding products to $M_{L}^{*}$ does not increase market shares, but strictly increases costs. Hence, the above is an equilibrium.

Suppose, by contradiction, that $\left(\left(M_{L}^{\prime}, m_{L}^{\prime}\right),\left(M_{F}^{\prime}, m_{F}^{\prime}\right)\right) \neq\left(\left(M_{L}^{*}, m_{L}^{*}\right),\left(M_{F}^{*}, m_{F}^{*}\right)\right)$ is another equilibrium. I have already examined the case in which $M_{L}^{*} \subset M_{L}^{\prime}$. Hence, suppose $M_{L}^{*} \not \subset M_{L}^{\prime}$. I distinguish two cases.

Case (i): at $\left(\left(M_{L}^{\prime}, m_{L}^{\prime}\right),\left(M_{F}^{\prime}, m_{F}^{\prime}\right)\right) F$ obtains no market shares. Hence, $F$ makes negative profits unless $M_{F}^{\prime}=\{(0, \ldots, 0)\}$. Since $M_{L}^{*} \not \subset M_{L}^{\prime}$, then $F$ has an incentive to deviate to $\left(M_{F}, j\right)$ such that $x \in M_{F}$, where $x \in M_{L}^{*} \backslash M_{L}^{\prime}$. By construction, $x$ is the $u$-highest utility alternative that possesses attribute $i$ for some $i \in\{1, \ldots, k\}$ and some $u \in U$. Let $j \equiv i$. Then at the deviation $F$ obtains at least $\frac{\text { Prob(type u) }}{2}$ market shares at a cost of at least $\sum_{x \in M_{F}} \sum_{i \in\{1, \ldots, k\}} x_{i}$. As long as costs are sufficiently small, the deviation is profitable, a contradiction.

Case (ii): at $\left(\left(M_{L}^{\prime}, m_{L}^{\prime}\right),\left(M_{F}^{\prime}, m_{F}^{\prime}\right)\right) F$ obtains some market shares. Then L has an incentive to deviate to $\left(M_{L}^{*}, m_{L}^{*}\right)$, as this strategy ensures that L obtains the whole 
market. If costs are sufficiently small, the deviation is profitable, which leads to a contradiction. Hence, there are no other equilibria.

Proposition 2 Assume that in equilibrium the consumer does not go through the noncompensatory phase $(N P)$. That is, $\left|M_{L} \cup M_{F}\right|=2$. I first show that such an equilibrium exists. Assume that there are two types $u$ and $v$ that occur with probability $\frac{1}{2}$. Assume that $\{x\}=\arg \max _{y \in\{0,1\}^{k}} u(y),\{z\}=\arg \max _{y \in\{0,1\}^{k}} v(y)$, and $\{x\}=$ $\arg \max _{y \in\{0,1\}^{k} \backslash\{z\}} v(y)$. Suppose that L chooses $\left(\{x\}, m_{L}\right)$ and $F\left(\{z\}, m_{F}\right)$, where $m_{L}$ is possessed by $x$ and $m_{F}$ by $z$ yielding $\frac{1}{2}$ market shares to each firm. I claim that $\left(\left(\{x\}, m_{L}\right),\left(\{z\}, m_{F}\right)\right)$ is an equilibrium. Suppose not. Then, there is a profitable deviation. Note that $L$ and $F$ cannot profitably deviate to a strategy $(\{w\}, m)$. If $F$ does so, then it obtains zero market shares. The same holds for L, unless L offers $\{z\}$ (at the deviation $F$ would respond with $x$ and $L$ would obtain exactly $\frac{1}{2}$ market shares), but the deviation would still not be profitable. Next, assume that assume that L deviates to $\left(M_{L}^{\prime}, m_{L}^{\prime}\right)$ such that $\left|M_{L}^{\prime}\right| \geq 2$. F's best response implies that $F$ can grab at least $\frac{1}{2}$ of the market shares by offering one alternative that possesses an attribute that the products in $M_{L}$ do not have and advertising that attribute. Hence, L obtains at most $\frac{1}{2}$ market shares at the deviation, but costs are at least as high. Finally, assume that $F$ deviates to a strategy $\left(M_{F}^{\prime}, m_{F}^{\prime}\right)$ such that $M_{L}$ is a doubleton menu. F can grab at most $\frac{1}{2}$ of the market shares, as even though there is some $t \in M_{F}^{\prime}$ such that $t$ possesses attribute $m_{L}$, $t$ is always beaten by $x$.

Next, I show that in any equilibrium both firms are active in the market. That is, $M_{i} \neq$ $\{(0, \ldots, 0)\}$ with $i \in\{L, F\}$. Suppose not. Assume first that $M_{L}=\{(0, \ldots, 0)\} \neq$ $M_{F}$. L can deviate to $\left(\{x\}, m_{L}\right)$ such that $m_{L}$ is possessed by $x$ and the probability that $x$ is a highest utility product is maximized. This deviation yields positive market shares, a contradiction. Next, assume that $M_{F}=\{(0, \ldots, 0)\} \neq M_{L} . F$ can deviate to $\left(\{z, t\}, m_{F}\right)$ such that $m_{F} \in \hat{I}_{M_{L}}, m_{F}$ is possessed by $z$, $z$ is a highest utility product with positive probability, and tis a cheapest alternative whose only purpose is to make the feasible set complex. This deviation yields positive market shares, a contradiction.

Assume that in equilibrium the consumer goes through the NC. An example of such an equilibrium is provided in the main body. I argue by backward induction that in any equilibrium in which the consumer goes through the NP marketing is relevant and firm-specific. Assume that L offers $\left(M_{L}, m_{L}\right)$. Then, $F$ advertises an attribute $m_{F} \in \hat{I}_{M_{L}}$ that the products offered by $L$ do not possess and offers an alternative that possesses $m_{F}$ and (if feasible) $m_{L}$. Denote the cheapest corresponding menu by $\left(M_{F}^{*}, m_{F}^{*}\right)$. This ensures that $F$ grabs at least $\frac{1}{2}$ market shares and competition occurs only in the state of the world in which the consumer's essential attribute is $m_{L}$.

Assume that $F$ chooses $\left(M_{F}^{*}, m_{F}^{*}\right)$. L's best response is as follows. First, fix a marketing strategy $m_{L}$ and a 'machinery' $\hat{I}_{M_{L}}$. Then, construct a menu $M_{L}\left(m_{L}, I_{M_{L}}\right)$ that contains all $u$-highest utility alternatives that possess attribute $m_{L}$. Second, identify the optimal machinery $\hat{I}_{M_{L}}^{*}$ that maximizes the probability that $x \in M_{L}\left(m_{L}, \hat{I}_{M_{L}}\right)$ is a u-highest utility alternative that possesses $m_{L}$ and denote the corresponding $M_{L}\left(m_{L}, \hat{I}_{M_{L}}^{*}\right)$ by $M_{L}\left(m_{L}\right)$. Third, select the optimal marketing strategy $m_{L}^{*}$ which maximizes the probability that products in $M_{L}\left(m_{L}\right)$ are chosen when the consumer's salient attribute is $m_{L}$ and denote the cheapest corresponding menu $M_{L}\left(m_{L}^{*}\right)$ by $M_{L}^{*}$. 
Independence of the Axioms. Let $x=(110), y=(101), z=(011)$, and $w=(001)$.

- $c$ satisfies all axioms, but A5. Let $X=\{x, y, z\}$ and assume that $x=c(\{x, y\})$, $x=c(\{x, z\}), y=c(\{y, z\})$, and $z=c(X)$.

- $c$ satisfies all axioms, but A4. Let $X=\{x, y, z\}$ and assume that $x=c(\{x, y\})$, $x=c(\{x, z\}), y=(c(\{y, z\})$, and $\emptyset=c(X)$.

- $c$ satisfies all axioms, but A3. Let $X=\{x, y, z\}$ and assume that $x=c(\{x, y\})$, $x=c(\{x, z\}), \emptyset=c(\{y, z\})$, and $x=c(X)$.

- $c$ satisfies all axioms, but A2. Let $X=\{x, y, w\}$ and assume that $x=c(\{x, y\})$, $x=c(\{x, z\}), w=c(\{y, w\})$, and $x=c(X)$.

- $c$ satisfies all axioms, but A1. Let $X=\{x, y, z\}$ and assume that $x=c(\{x, y\})$, $z=c(\{x, z\}), y=c(\{y, z\})$, and $x=c(X)$.

Proposition 3 Necessity. Assume that c is a CLT. A1-A3 are clearly satisfied. Assume, by contradiction, that $A 4$ is violated. Then, $c(A)=\emptyset$ implies that $A(i) \neq\{\emptyset\}$ for all $i \in I$. Let $i_{A}$ be any attribute in I. Then, $\emptyset \notin \Phi(A)$ implying that $c(A) \neq \emptyset, a$ contradiction.

Next, assume, by contradiction, that $A 5$ is violated. Then, $A(i) \cap U C_{x, A} \neq \varnothing$ for any $i \in I(x)$ implies that $x=c(A)$. If $i_{A} \notin I(x)$, the contradiction arises immediately. Hence, assume that $i_{A} \in I(x)$. Then, $x \in \Phi(A)$. However, since $A(i) \cap U C_{x, A} \neq \varnothing$ for any $i \in I(x)$, then $\exists y \in \Phi(A)$ such that $u(y)>u(x)$. This implies that $x \notin$ $\arg \max _{z \in \Phi(A)} u(z)$ and consequently $x \neq c(A)$, which leads to a contradiction.

Sufficiency. Assume that $c$ satisfies A1-A5. By A3 the choice function is never empty at binary choice problems. Let $x \rightarrow y$ whenever $x=c(\{x, y\})$ be the revealed preference relation. By $A 1$ and standard arguments, $\rightarrow$ is a strict rational preference relation. Since $c$ satisfies $A 2$, then $x \rightarrow y$ whenever $I(y) \subset I(x)$. Let $u:\{0,1\}^{k} \cup$ $\{\emptyset\} \rightarrow \Re$ be such that $u(x)>u(y)$ whenever $x \rightarrow y$ and $\{\emptyset\}=\arg \min _{y \in X \cup\{\emptyset\}} u(y)$.

Let $A \in \mathcal{D}$ be any choice problem. Let $c_{C L T}(A) \equiv \arg \max _{y \in \Phi(A)} u(y)$ for some $\Phi(A) \subseteq A \cup\{\emptyset\}$. I want to show that $c(A)=c_{C L T}(A)$. I distinguish two cases.

Case $(i):|A|=2$. Let $\Phi(A)=$ A. Assume that $x=c(A)$ and suppose, by contradiction, that $x \neq c_{C L T}(A)$. Then, $u(y)>u(x)$, where $y$ is the unique alternative in $\Phi(A) \backslash\{x\}$. However, the fact that $x=c(A)$ implies, by construction, that $u(x)>u(y)$, a contradiction. Hence, $c(A)=c_{C L T}(A)$.

Case (ii): $|A|>2$. Let $\Phi(A) \equiv A\left(i_{A}\right)$ for some $i_{A} \in I$. I distinguish two sub-cases.

Sub-case $(a): c(A)=\emptyset$. By $A 4, A(j)=\{\emptyset\}$ for some $j \in I$. Let $i_{A}=j$. Then, $\Phi(A)=\{\emptyset\}$ and consequently $c_{C L T}(A)=\emptyset$, as desired

Sub-case $(b): x=c(A) \neq \emptyset$. By A5, there exists $j \in I(x)$ such that $A(j) \cap U C_{x, A}=$ Ø. Let $i_{A}=j$. Then, $\Phi(A) \cap U C_{x, A}=\emptyset$ implying that $x$ is the highest utility alternative in $\Phi(A)$. Hence, $c_{C L T}(A)=x$, as desired.

Proposition 4 Necessity. Assume first that $c$ is a CLT*. Since $i_{A} \in I\left(x^{* A}\right)$ for any $A \in \mathcal{D}$, where $\left\{x^{* A}\right\}=\arg \max _{y \in A \cup\{\emptyset\}} u(y)$, then $x^{* A} \in \Phi(A)$ for any $A \in \mathcal{D}$. This implies that $\{c(A)\}=\arg \max _{y \in A \cup\{\emptyset\}} u(y) \neq \emptyset$. Let $x>y$ if and only if $u(x)>u(y)$ be the preference induced by the utility function. Note that $\succ$ is a strict rational preference relation. Then, at any $A \in \mathcal{D}, c(A)$ is the $\succ$-maximal element of $A$. By Arrow (1959), c satisfies WARP. 
Sufficiency. In the other direction, assume that c satisfies WARP. Since attributes are good, then c satisfies A2. Next, since $\mathcal{D}$ contains all subsets of $X$ up to three elements and the choice function is never empty, then, by Arrow (1959), $\{c(A)\}=\max (A ; \succ)$ for some strict rational preference $\succ$. Since c satisfies A2, then $I(y) \subset I(x)$ implies that $x>y$. Let $u(x)>u(y)$ if and only if $x>y$ and $\{\emptyset\}=\arg \min _{y \in X \cup\{\emptyset\}} u(y)$. For every $A \in \mathcal{D}$, let $i_{A} \in I\left(x^{* A}\right)$, where $\left\{x^{* A}\right\}=\arg \max _{y \in A \cup\{\emptyset\}} u(y)$. Let $c_{C L T^{*}} \equiv$ $\arg \max _{y \in \Phi(A)} u(y)$, where $\Phi(A)$ is equal to $A\left(i_{A}\right)$ if $|A|>2$ and to $A$, otherwise. I

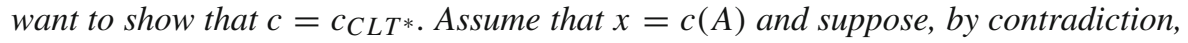
that $x \neq c_{C L T^{*}}(A)$. Then, $u(y)>u(x)$ for some $y \in A \backslash\{x\}$ or, equivalently, $y \succ x$ for some $y \in A \backslash\{x\}$. Hence, $x \notin \max (A ; \succ)$. However, this implies that $x \neq c(A), a$ contradiction.

\section{References}

Armstrong, M.: Interactions between competition and consumer policy. Compet. Policy Int. 4, 97-147 (2008)

Arrow, K.J.: Choice functions and orderings. Economica 26(102), 121-127 (1959)

Bordalo, P, Gennaioli, N, Shleifer, A.: Competition for attention. Mimeo (2013)

Dahremoeller, C., Fels, M.: Product lines, product design, and limited attention. Mimeo (2013)

Eliaz, K., Spiegler, R.: Consideration sets and competitive marketing. Rev. Econ. Stud. 78, 235-262 (2011)

Eliaz, K., Richter, M., Rubinstein, A.: Choosing the two finalists. Econ. Theory 46, 211-219 (2011)

Ellison, G.: Bounded rationality in industrial organization. In: Blundell, R. Newey, W., Persson, T. (eds.) Advances in Economics and Econometrics: Theory and Applications. Cambridge University Press, Cambridge (2006)

Gigerenzer, G., Hertwig, R., Pachur, T.: Heuristics. The Foundations of Adaptive Behavior. Oxford University Press, Oxford (2011)

Hauser J.R., Ding, M., Gaskin, S.P.: Non-compensatory (and compensatory) models of consideration-set decisions. In: Proceedings of the Sawtooth Software Conference, Delray, FL, pp. 207-232 (2009)

Manzini, P., Mariotti, M.: Sequentially rationalizable choice. Am. Econ. Rev. 97(5), 1824-1839 (2007)

Masatlioglu, Y., Nakajima, D., Ozbay, E.Y.: Revealed attention. Am. Econ. Rev. 102(5), 2183-2205 (2012)

Payne J.W., Bettman, J.R., Johnson, E.J.: The Adaptive Decision Maker. Cambridge University Press, Cambridge (1993)

Piccione, M., Spiegler, R.: Price competition under limited comparability. Q. J. Econ. 127, 97-135 (2012)

Spiegler R.: Bounded Rationality and Industrial Organization. Oxford University Press, Oxford (2011)

Spiegler, R.: Monopoly pricing when consumers are antagonized by unexpected price increases: a 'Cover Version' of the Heidhues-Koszegi-Rabin model. Econ. Theory 51, 695-711 (2012)

Spiegler, R.: Competitive framing. AEJ Micro (2013) (forthcoming) 